\title{
Control rhythm rather than rate to delay permanent AF
}

The choice of management strategy in patients with recent-onset AF does not affect clinical outcomes, but does influence the likelihood of progression to permanent AF, according to data from an international registry of patients with arrhythmias. Patients with AF usually receive antithrombotic treatment, and a strategy to control either rate (by keeping ventricular rate at a physiological level, while allowing continued atrial flutter) or rhythm (by restoration and maintenance of sinus rhythm) to manage recurrent arrhythmic episodes.

Data on 5,604 individuals with AF (3,076 on rhythm-control and 2,528 on rate-control treatment) from 21 countries across Europe, America, and Asia were included in the registry. For rhythm control, successful treatment was defined as the patient being in sinus rhythm on the electrocardiogram at the final (12-month) visit. Rate control was deemed a success if the patient's resting heart rate was $\leq 80 \mathrm{bpm}$ on the electrocardiogram. The criteria for therapeutic success also required there to have been no cross-over between management strategies, and for no clinical outcomes to have occurred between the baseline and final visits.

At the 1 year follow-up, $92.3 \%$ of patients were assessable, and 54\% of patients fulfilled the criteria for successful treatment $(60 \%$ and $47 \%$ of rhythmcontrolled and rate-controlled patients, respectively). The rhythm-control strategy was significantly related to superior therapeutic success (odds ratio 1.34, 95\% CI $1.15-1.55, P=0.0002)$. At the time of their follow-up electrocardiogram, $80.5 \%$ of patients managed with a rhythm-control strategy were in sinus rhythm, compared with only $33.2 \%$ of rate-controlled patients. Furthermore, evolution to permanent AF occurred in $13.3 \%$ of rhythm-controlled patients, and $53.8 \%$ of rate-controlled patients, indicating that rhythm control significantly reduces the likelihood of AF progression (odds ratio $0.20,95 \%$ CI $0.17-0.25, P<0.0001)$.
However, clinical outcomes in patients with AF were driven predominantly by hospitalizations and other cardiovascular causes, rather than choice of AF-management strategy.

This study was limited by the fact that patients were not randomly allocated to management strategy, so the two groups differed greatly in patient characteristics. Additionally, the final assessment of therapeutic success was on the basis of a single electrocardiogram recording, which is likely to be an inadequate indication of the frequency of AF. Nonetheless, this 'real-world' study strongly indicates that rhythm control is a superior strategy to rate control for the prevention of progression to permantent AF.

Gregory B. Lim

Original article Camm, A. J. et al. Real-life observatiions of clinical outcomes with rhythm- and rate-control therapies for atrial fibrillation: RECORDAF (Registry on Cardiac Rhythm Disorders Assessing the Control of Atrial Fibrillation) J. Am. Coll. Cardiol. 58, 493-501 (2011) 